\title{
MODELING TIME-DEPENDENT DEFORMATIONS OF CONCRETE MEMBERS SUBJECTED TO SYMMETRIC AND ASYMMETRIC DRYING
}

\author{
Pavel Horák*, Petr Havlásek \\ Czech Technical University in Prague, Faculty of Civil Engineering, Department of Mechanics, Thákurova \%, \\ 16629 Prague 6, Czech Republic \\ * corresponding author: pavel.horak@fsv.cvut.cz
}

\begin{abstract}
The time-dependent behavior of concrete members with uniform cross-section subjected to symmetric drying can be predicted with one of many models anchored in the design codes. Since these models use the cross-sectional approach, only the average quantities can be obtained. More complex phenomena can be investigated by means of a coupled hygro-mechanical finite element analysis. Owing to insufficient or missing experimental data, the input parameters for the moisture transport model are often tuned to match the development of axial shrinkage or moisture loss measured on small-scale laboratory specimens. In this paper it is assessed whether this approach leads to a unique set of parameters of the Bažant-Najjar model for moisture diffusion. Additionally, for the selected sets of parameters the analysis is repeated with modified size and/or boundary conditions leading to asymmetric drying and the results are discussed.
\end{abstract}

KEYWORDs: Concrete, drying shrinkage, finite elements, moisture transport, parameter identification.

\section{INTRODUCTION AND MOTIVATION}

Creep and shrinkage are complex time-dependent phenomena in concrete whose magnitude and evolution is influenced by many factors. The distinction between creep and shrinkage, which are both related to delayed strains, is that creep is caused by loading. At constant temperature the creep deformation can be additively decomposed into basic creep and drying creep. Basic creep is the deformation of hygrally sealed concrete while drying creep is an additional deformation caused by drying. In a similar fashion, autogenous and drying shrinkage refer to the volume change (typically contraction) of sealed and drying concrete, respectively. In normal-strength structural concretes with $w / c \geq 0.4$, the autogenous shrinkage is typically negligible $\left(\approx 40 \times 10^{-6}\right)$ in comparison to the magnitude of drying shrinkage $\left(\approx 780 \times 10^{-6}\right.$ at $40 \%$ relative humidity according to ACI 209).

This time-dependent behavior of typical concrete members can be estimated using the prediction models (e.g. B3, B4, fib Model Code) or the formulae from the design codes (e.g. ACI 209, Eurocode 2). However, since these models operate on the cross-sectional level, only the average quantities can be obtained. In order to describe more complex phenomena, e.g. tensile cracking produced by stress gradients caused by nonuniform drying or the structural response to non-symmetric drying, a more versatile approach is a necessity.

One of the options is a coupled hygro-mechanical finite element analysis. The bottleneck of this approach resides in often insufficient number of experiments which are necessary for proper calibration of the complex constitutive models. To overcome this obstacle, certain parameters are set to their recommended values or the parameters are adjusted to reproduce the observed macroscopic behavior. To illustrate this, in the recent paper [1], this approach was used by several research groups to calibrate the transport model based on the axial shrinkage and moisture loss of a standard laboratory specimen without any information on the distribution of relative humidity.

The primary objective of this paper is to assess whether such approach yields a unique set of material parameters and what macroscopic measurements can narrow the range of the identified values.

\section{Methodology}

All results presented further in this paper were obtained using a one-way coupled hygro-mechanical simulations in open-source finite element package OOFEM [2]. The constitutive models described in more detail in the following sections were calibrated on creep and shrinkage data reported by Bryant [3]. The parameters of the structural material model are then fixed while the parameters in the model for moisture transport are subject to optimization under various assumptions. The identified parameters are consequently utilized for predicting the curvature of an infinite slab subjected to asymmetric drying.

\subsection{MATERIAL MODELS}

Concrete drying is in the present study described by a widely recognized model proposed by Bažant and Najjar [4]. The governing equation for the diffusion of water vapor was derived under the assumption of 
constant moisture capacity $k$ (slope of the desorption isotherm). When the moisture sink is neglected the equation reads

$$
\frac{\partial h}{\partial t}=\nabla \cdot(C(h) \nabla h)
$$

where $\nabla h$ is the gradient of relative humidity and $C(h)$ is the humidity-dependent diffusivity. This dependence is for the cementitious materials highly nonlinear and can be approximated as

$$
C(h)=C_{1}\left(\alpha_{0}+\frac{1-\alpha_{0}}{1+\left(\frac{1-h}{1-h_{c}}\right)^{n}}\right)
$$

where $C_{1}$ is the maximum diffusivity at $h=1, \alpha_{0}$ determines the ratio between minimum diffusivity at $h=0$ and $C_{1}$, and parameters $h_{c}$ and $n$ describe the relative humidity and the rate of the transition. This model was embedded into the $f i b$ Model Code 2010 [5] which recommends the following values $\alpha_{0}=0.05, h_{c}=0.8, n=15$, while $C_{1}$ is to be determined from the compressive strength. In the simulations, the initial value of relative humidity is set to $h=0.98$ which is the typical value for the sealed conditions and normal-strength concrete with negligible self-desiccation. The ambient relative humidity is idealized as constant and is prescribed using the mixed boundary condition which uses the surface factor $f$ to relates the humidity flux $J_{h}$ with the difference between the relative humidity at the boundary and the environment

$$
J_{h}=f \cdot\left(h_{e n v}-h\right)
$$

In each time step the moisture transport is followed by the structural sub-problem which utilizes the computed field of relative humidity. The constitutive vicoelastic model is the modified [6] Microprestresssolidification (MPS) theory [7]. Under constant temperature and hygrally sealed conditions the material model reduces to the B3 model [8]. Changes in relative humidity give rise to additional compliance, referred to as drying creep or the Pickett effect, and to volume changes-shrinkage or swelling. The model has been modified to minimize the size-effect on drying creep which is controlled by parameter $k_{3}$. Shrinkage strain and relative humidity are linearly linked via their rates,

$$
\dot{\varepsilon}_{s h}=k_{s h} \dot{h}
$$

where $k_{s h}$ is a material parameter usually treated as humidity- and age-independent constant. The model can be enhanced by tensile softening but this feature is not activated in the present study.

\subsection{Modeling Strategy}

Despite the unidirectional nature of the transport problem, the analysis was performed in two dimensions to provide the subsequent structural analysis with the distribution of relative humidity. The mechanical problem was carried out under the assumption of plane stress which allowed to run the computations very efficiently and repeatedly as described later.

The assumption of plane stress is very realistic in the case of the experimental data. The parametric study is done on an idealized plane section cut out from an "infinite slab" drying from one or two surfaces. Yet, the model which is hereafter referred to as "slab", neglects the stresses perpendicular to the section and its behavior corresponds to a beam with prismatic cross-section, sealed lateral sides and drying from top and/or bottom surfaces. The effect of self weight is omitted in the study. The finite element meshes of the two sub-problems were identical and were composed of 60 quadrilateral elements with linear approximation of humidity and displacement. The meshes were refined near the drying surfaces. All problems were solved in 284 time steps with increasing length.

As mentioned earlier, the material parameters were calibrated on experimental data by Bryant and Vahanavikkit [3]. Their study was focused on creep and shrinkage of structural concrete $\left(f_{c}=50.1 \mathrm{MPa}\right.$, $w / c=0.47)$ measured on prismatic members with cross-section ranging from $100 \times 100$ to $400 \times 400 \mathrm{~mm}$. The specimens were either entirely sealed to investigate basic creep or partially sealed to study drying creep and shrinkage. Drying at $60 \%$ relative humidity began at the age of 8 days. The majority of the creep tests imposing $7 \mathrm{MPa}$ compression started at the age of 14 days. Additional tests were done to identify aging. All loaded specimens had a companion without external loading. The constant room temperature was maintained throughout the experiment.

Calibration began with the basic creep compliance function. The parameters $q_{1}-q_{4}$ of the B3 model were adopted from [9] and their values were $q_{1}=9$, $q_{2}=75, q_{3}=28$ and $q_{4}=6.5$ all in $10^{-6} / \mathrm{MPa}$. Drying creep and shrinkage was calibrated on experimental data of prism $150 \times 150 \mathrm{~mm}$ drying from two lateral sides (i.e. $150 \mathrm{~mm}$ thick infinite wall). This specimen is the largest one which has reached the ultimate values and for this reason was selected as reference. With crudely calibrated parameters of the transport model the shrinkage constant was identified as $k_{s h}=1.9 \times 10^{-3}$, and drying creep parameter $k_{3}=25$.

The parameters of the Bažant-Najjar model and the surface factor were identified using a brute-force method implemented in a computer program. The repeatedly run analysis used the values with the ranges and stepping summarized in Table 1 which makes altogether 31360 combinations. The accuracy of the fit was assessed by means of a weighted error which considered the drying period on the log scale. The reference set of parameters which gave the best fit of with experimental data is listed in Table 1 (see solid 


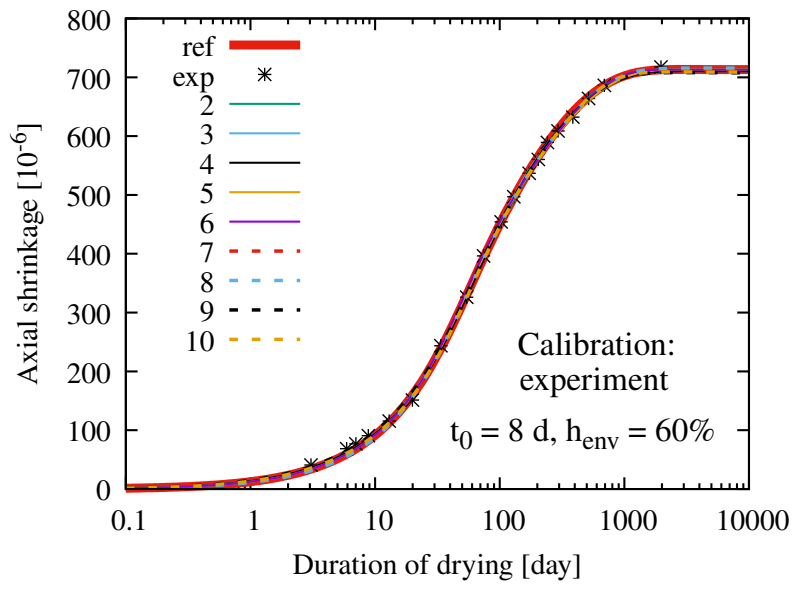

Figure 1. Best fits of axial shrinkage measured by Bryant and Vadhanavikkit [3] on $150 \mathrm{~mm}$ thick slabs, $t_{0}=8$ days, $h_{\mathrm{env}}=60 \%$.

red line in Figs. 1 and 2). This set was later used to define the reference behavior under a modified setup (i.e. different size or boundary conditions).

In the parametric study the analysis starts at the age of 28 days and the ambient relative humidity is set to $h_{\text {env }}=50 \%$ with the intention to minimize the effect of aging and to comply with the standard laboratory conditions. Furthermore, as demonstrated in the following section, it was attempted to narrow the identified set of parameters by incorporating additional reference data: moisture loss (assuming $k=100 \mathrm{~kg} / \mathrm{m}^{3}$ ), response to drying at different levels of relative humidity (30\% and 70\%) and shrinkage of a symmetrically drying slab with different thickness (75 $\mathrm{mm}$ and $300 \mathrm{~mm}$ ). Moreover, a model of $75 \mathrm{~mm}$ thick slab drying from one surface only is used to assess the influence of the identified diffusivity functions on the structural behavior. This model has the same effective thickness (and thus drying kinetics) but turns out to be significantly more sensitive to the shape of the diffusivity function.

\begin{tabular}{lccccc}
\hline & $\begin{array}{c}C_{1} \\
{\left[\mathrm{~mm}^{2} / \text { day }\right]}\end{array}$ & $\begin{array}{c}\alpha_{0} \\
{[-]}\end{array}$ & $\begin{array}{c}h_{c} \\
{[-]}\end{array}$ & $\begin{array}{c}n \\
{[-]}\end{array}$ & $\begin{array}{c}f \\
{[\mathrm{~mm} / \text { day }]}\end{array}$ \\
\hline min. & 35 & 0.02 & 0.68 & 6 & 1.1 \\
max. & 80 & 0.23 & 0.80 & 20 & 2.3 \\
step & 5 & 0.03 & 0.02 & 2 & 0.2 \\
ref. & 60 & 0.05 & 0.74 & 0.06 & 1.5 \\
\hline
\end{tabular}

TABLE 1. Parameters of the transport problem: ranges, stepping for identification and the reference set.

\section{Results AND Discussion}

The solution obtained with the reference set of material parameters (Tab. 1) is in the following figures shown consistently in thick red lines. As demonstrated in Fig. 1, the axial shrinkage measured by

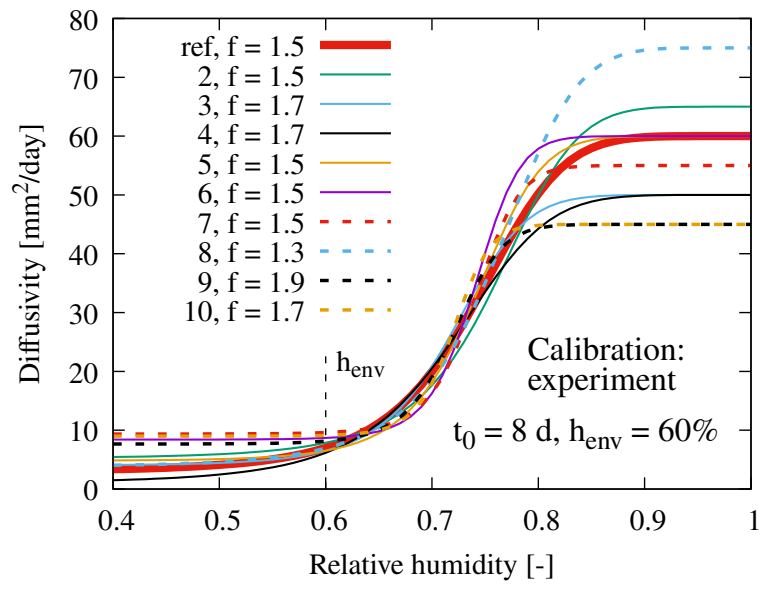

Figure 2. Dependence of diffusivity on relative humidity for the 10 best fits in Fig. 1 . In the legend $f$ is the surface factor $[\mathrm{mm} /$ day].

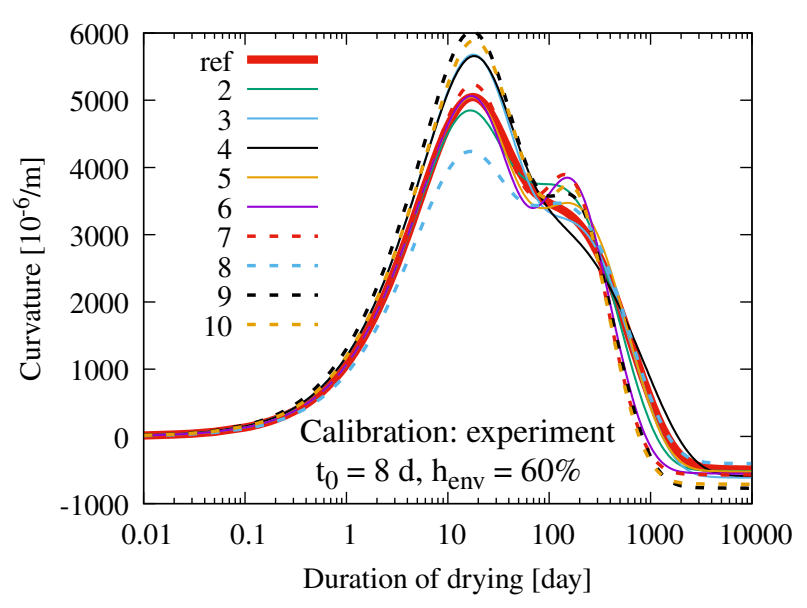

Figure 3. Prediction of curvature of $75 \mathrm{~mm}$ thick slab exposed to drying from one side only at $h_{\mathrm{env}}=$ $50 \%$ which started at the age $t_{0}=28$ days. The line types and the material parameters correspond to Figs. 1 and 2.

Bryant on prism $150 \times 150 \mathrm{~mm}$ drying from two opposite surfaces is with very similar accuracy captured by numerous combinations of material parameters of the diffusivity function. Figure 1 presents only the 10 best fits, the respective functions are shown in Fig. 2 and the legend lists the values of the surface factor $f[\mathrm{~mm} /$ day $]$. From the fits, it can be concluded that the evolution of axial shrinkage is almost insensitive to the diffusivity at high relative humidity $(h>0.8)$ where the values span from 45 to $75 \mathrm{~mm}^{2}$ /day, but becomes very sensitive to the diffusivity in the region just above the ambient relative humidity $h_{\mathrm{env}}$ where the functions form a narrow band.

The response of a slab with the same effective thickness exposed to drying from one side only at standard laboratory conditions $\left(t_{0}=28\right.$ days, $\left.h_{\mathrm{env}}=50 \%\right)$ is for the best 10 identified combinations of diffusivity shown in Fig. 3. In contrast to symmetric drying, the computed response for asymmetric drying be- 


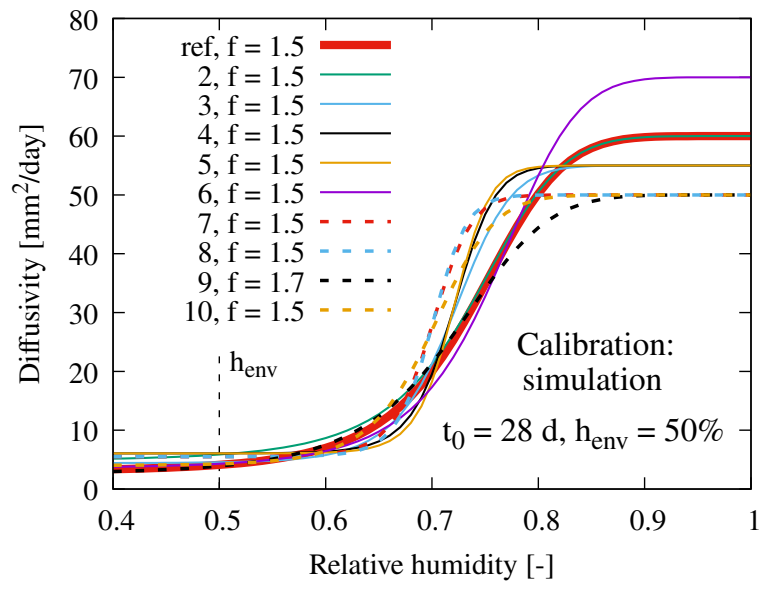

Figure 4. Diffusivity functions giving the best agreement with the axial shrinkage from the simulation of symmetrically drying concrete slab $(D=150 \mathrm{~mm}$, $h_{\mathrm{env}}=50 \%, t_{0}=28$ days) computed with the reference set of material parameters.

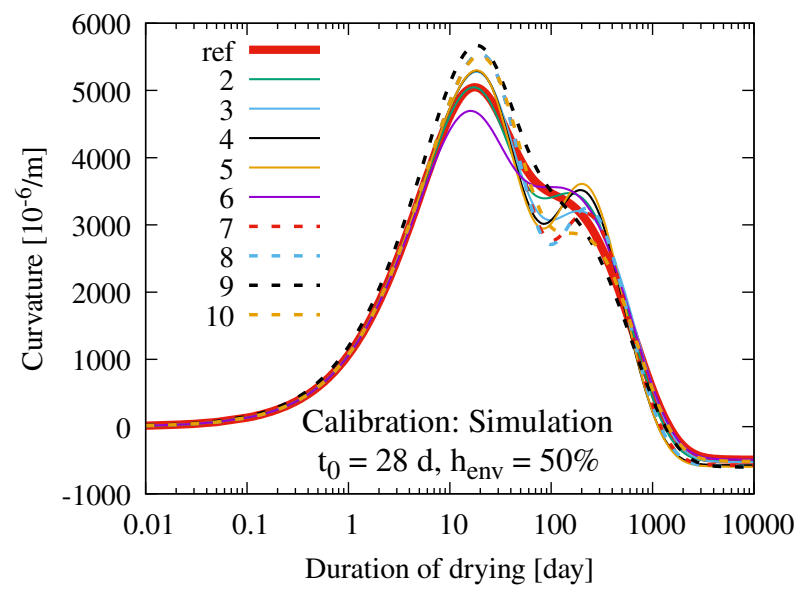

Figure 5. Prediction of curvature of $75 \mathrm{~mm}$ thick slab exposed to drying from one side only at $h_{\mathrm{env}}=$ $50 \%$ and at the age $t_{0}=28$ days. The line types and the material parameters correspond to Fig. 4.

comes surprisingly different. In all presented cases, the peak curvature is reached after $\approx 20$ days of drying, and the individual solutions differ by up to $20 \%$ of the reference solution. In several cases the curvature exhibits a second local maximum one order of magnitude later $(\approx 200$ days). The value at the first peak is tightly connected with the diffusivity at high relative humidity, the higher this diffusivity, the lower the curvature and vice versa (compare e.g. the dashed blue and black lines \#8 and \#9). The higher this diffusivity the lower is the gradient of relative humidity between the sealed and drying surface of the cross-section and thus the lower is the resulting curvature. The second peak appears when the diffusivity function has a steep transition between high and low relative humidity which is controlled by parameter $n$ in (2).

The preceding observations are confirmed in Fig-

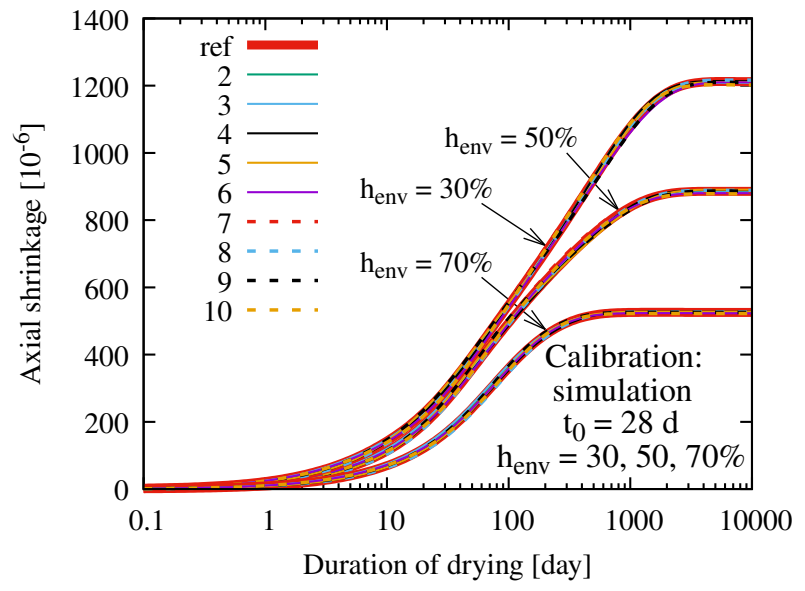

FIGURE 6. Best fits of (reference) axial shrinkage caused by symmetric drying at three levels of ambient relative humidity $h_{\mathrm{env}}=30,50,70 \%(D=150 \mathrm{~mm}$, $t_{0}=28$ days).

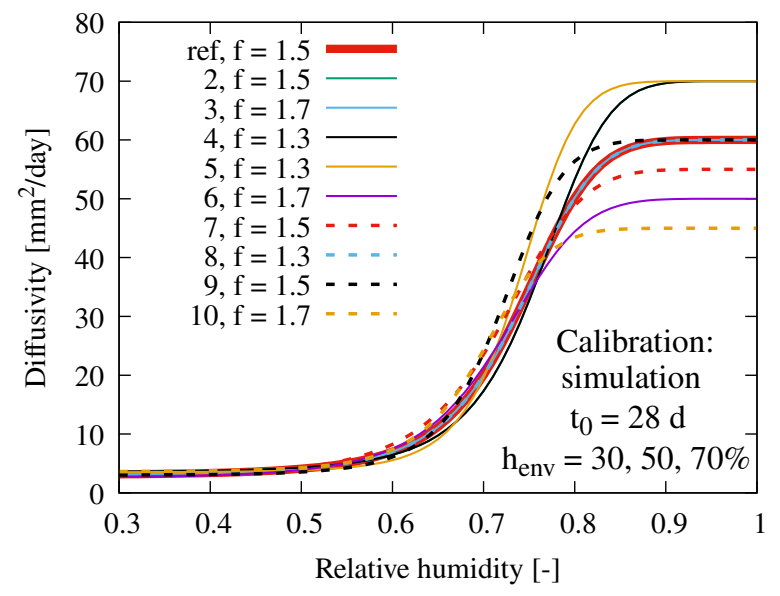

FiguRE 7. Diffusivity functions giving the best agreement with the axial shrinkage from the reference simulations (symmetric drying at three levels of ambient relative humidity $h_{\mathrm{env}}=30,50,70 \%, D=150 \mathrm{~mm}$, $t_{0}=28$ days).

ures 4 and 5 where the calibration was done on the data from simulation with reference parameters but at "laboratory conditions" $\left(t_{0}=28\right.$ days, $h_{\text {env }}=$ $50 \%$ ). The identified diffusivity functions are very close to the reference case in the region from $h_{\mathrm{env}}$ to $h_{\text {env }}+10 \%-15 \%$, while beyond this limit the functions are distinctively different which has an impact on the evolution of curvature.

Incorporating weight loss does not narrow the scatter of the identified diffusivity functions (figures are not presented here).

From the findings presented above it is natural to expect that if the separate measurements of axial shrinkage are performed at different levels of relative humidity, then the identified diffusivity functions will be closer to each other in the relative humidity slightly above the prescribed levels. To validate this statement, 2 additional values of relative humidity 


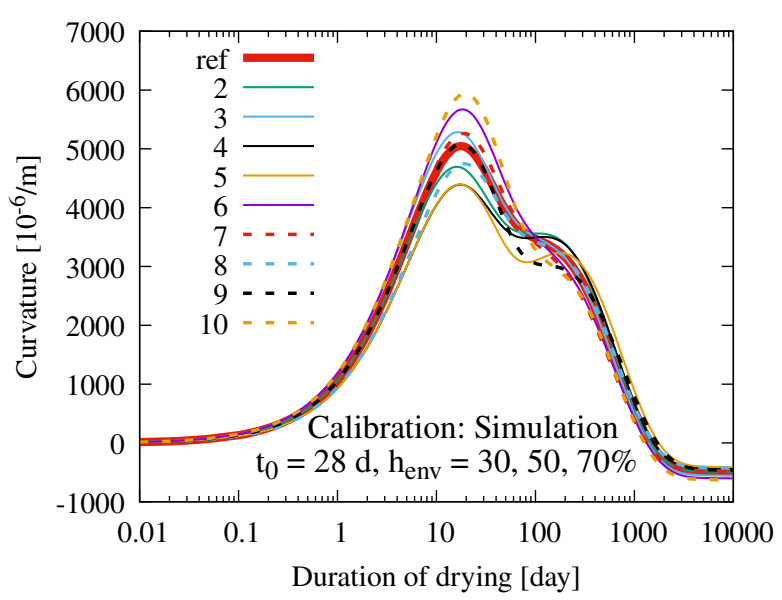

FiguRE 8. Prediction of curvature of $75 \mathrm{~mm}$ thick slab exposed to drying from one side only at $h_{\mathrm{env}}=$ $50 \%$ which started at $t_{0}=28$ days. The line types and the material parameters correspond to identification at 3 different levels of $h_{\text {env }}$ (Figs. 6 and 7).

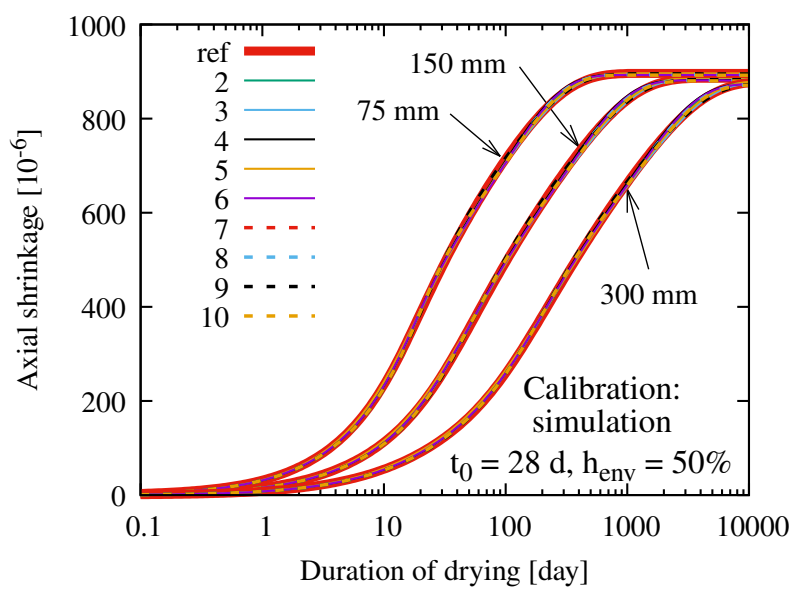

Figure 9. Best fits of (reference) axial shrinkage of $75 \mathrm{~mm}, 150 \mathrm{~mm}$, and $300 \mathrm{~mm}$ thick slabs exposed to symmetric drying at $h_{\mathrm{env}}=50 \%\left(t_{0}=28\right.$ days $)$.

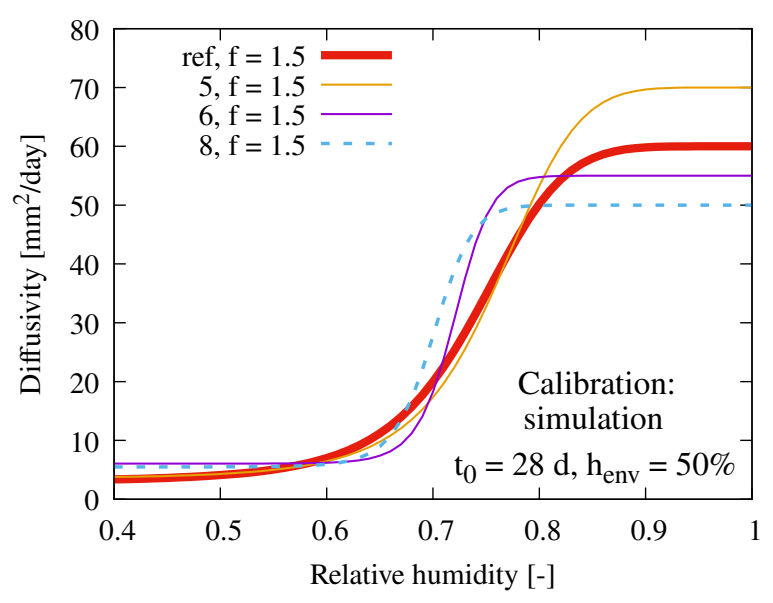

Figure 10. Diffusivity functions giving the best agreement with the reference simulations of axial shrinkage of symmetrically drying slabs with various thicknesses $\left(h_{\mathrm{env}}=50 \%, t_{0}=28\right.$ days, Fig. 9$)$.

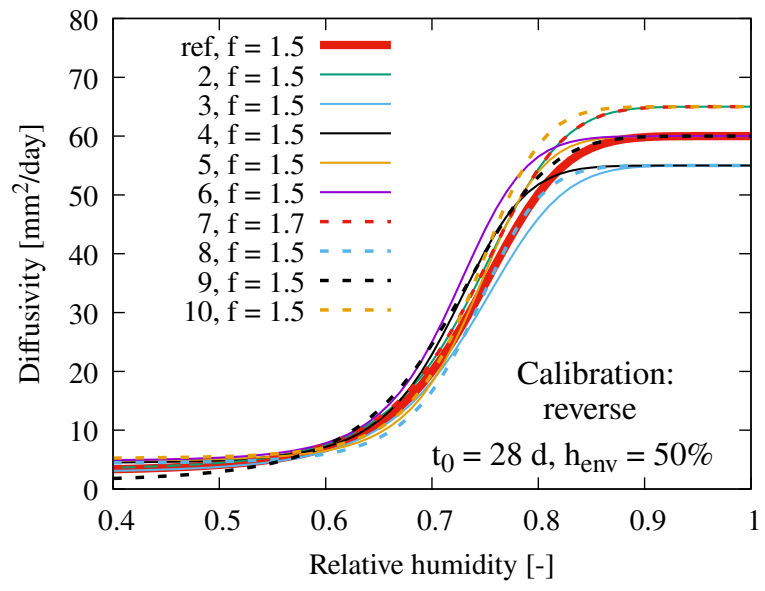

FiguRE 11. Diffusivity functions calibrated inversely on the evolution of curvature of $75 \mathrm{~mm}$ thick slab drying from one side $\left(h_{\mathrm{env}}=50, t_{0}=28\right.$ days $)$ obtained from the simulation with the reference set of material parameters.

were added and the analysis was done at 30\%, 50\% and $70 \%$. The 10 best fits are almost indistinguishable from the reference solution of axial shrinkage (see Fig. 6) and indeed, the identified diffusivity functions form a narrow band which widens with increasing relative humidity (Fig. 7).

This has a direct impact on the computed curvature shown in Fig. 8 where the scatter in the first peak persists (caused by high scatter of the maximum diffusivity), but beyond 100 days of drying the agreement between the solutions becomes significantly better.

Next, it was attempted to identify the diffusivity function based on the shrinkage evolution of specimens (i.e. models) with different effective sizes. The reference thickness $D=150 \mathrm{~mm}$ was doubled to $300 \mathrm{~mm}$ and reduced to $75 \mathrm{~mm}$. The 10 best fits match the solution with reference parameters perfectly (Fig. 9), yet the diffusivity functions show considerable scatter, see Fig. 10. Also it needs to be emphasized that such approach can turn out to be very demanding on the experimental duration since the evolution scales with $D^{2}$.

To summarize, and as illustrated in Figs. 12 and 13 it turned out that the shrinkage of symmetrically drying specimen is significantly less sensitive to the diffusivity function compared to the curvature produced by non-symmetric drying. For this reason the diffusivity identified from axial shrinkage should be used with caution and to members with similar size and boundary conditions. On the other hand the diffusivity identified from the evolution of curvature (see Fig. 11) exhibits significantly less scatter and so does the back-calculated axial shrinkage which matches the reference case perfectly (not presented here). 


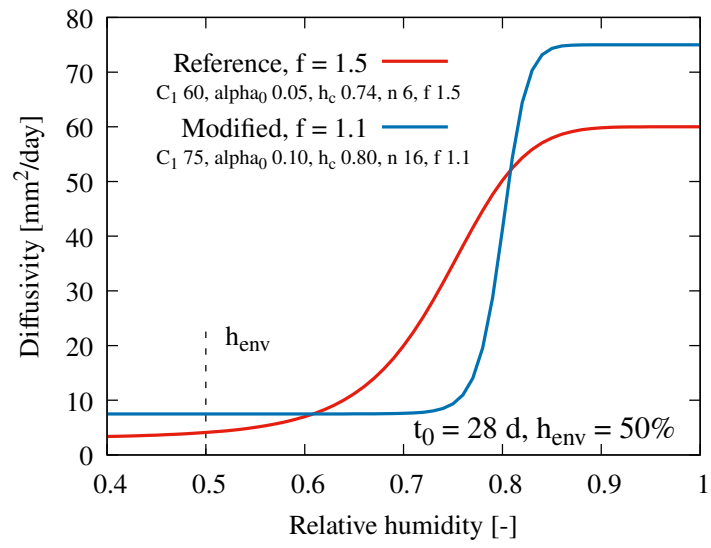

FiguRE 12. Diffusivity function with the "reference" and "modified" set of material parameters.

\section{Conclusions}

This paper investigated drying shrinkage of concrete slabs exposed to symmetric and asymmetric drying at constant ambient relative humidity. The problem was approached by means one-way coupled finite element simulations in program OOFEM. The moisture transport was described using the the Bažant-Najjar model while the time-dependent behavior of concrete by the modified MPS model. The emphasis was on put on identification of material parameters of the model for moisture transport and their uniqueness. The following conclusions can be drawn from the analysis:

- Identification of parameters based the evolution of axial shrinkage of symmetrically drying concrete does not yield unique set of material parameters. The reference data could be reproduced with various sets of parameters with accuracy similar or better than the typical experimental scatter.

- Incorporating the shrinkage data measured at different levels of relative humidity helps to narrow the identified diffusivity functions. This is not the case of the data on moisture loss or shrinkage of specimens with different size.

- The behavior of concrete drying non-symmetrically is considerably more sensitive to the values of material parameters. The parameters identified from the evolution of curvature give more consistent diffusivity functions and result into almost identical axial shrinkage.

- The presence and the character of the second maximum in the evolution of curvature is most sensitive to the exponent $n$ which defines the steepness of the transition between small and high diffusivity.

- The numerical simulations of concrete drying from one side only exhibit very little sensitivity to concrete creep (Fig. 13).

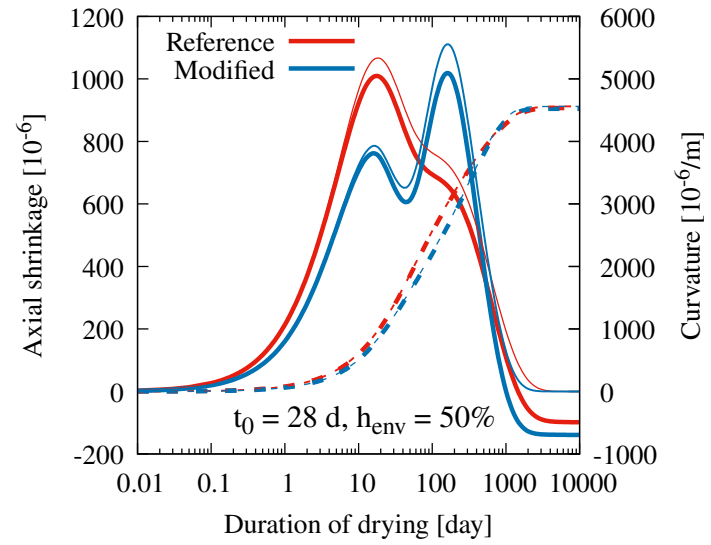

FiguRE 13. Evolution of axial shrinkage and curvature obtained with the "reference" and "modified" set of material parameters of the diffusivity function (Fig. 12) and $D=150 \mathrm{~mm}, t_{0}=28$ days, $h_{\mathrm{env}}=50 \%$. Thin lines correspond to the behavior without viscoelastic effects.

\section{ACKNOWLEDGEMENTS}

The authors gratefully acknowledge financial support from the Grant Agency of the Czech Technical University in Prague, project number SGS20/038/OHK1/1T/11, and from the Czech Science Foundation (GA ČR), project number 19-20666S.

\section{REFERENCES}

[1] V. Šmilauer, P. Havlásek, T. Gasch, et al. Hygro-mechanical modeling of restrained ring test: COST TU1404 benchmark. Construction and Building Materials 229:116543, 2019.

DOI:https://doi.org/10.1016/j.conbuildmat.2019.07.269.

[2] B. Patzák. OOFEM home page, 2000. http: //www . oof em.org.

[3] A. H. Bryant, C. Vadhanavikkit. Creep, shrinkage-size, and age at loading effects. ACI Materials Journal 84:117-123, 1987.

[4] Z. P. Bažant, L. J. Najjar. Nonlinear water diffusion in nonsaturated concrete. Materials and Structures 5:3-20, 1972. DOI:10.1007/BF02479073.

[5] Fédération Internationale du Béton. Model Code 2010. No. vol. 65 in fib Bulletin. International Federation for Structural Concrete (fib), 2012.

[6] Z. Bažant, P. Havlásek, M. Jirásek. Microprestress-solidification theory: Modeling of size effect on drying creep. In N. Bicanic, H. Mang, G. Meschke, R. de Borst (eds.), Computational Modelling of Concrete Structures, pp. 749-758. CRC Press/Balkema, EH Leiden, The Netherlands, 2014.

[7] Z. P. Bažant, A. P. Hauggaard, S. Baweja, F. J. Ulm. Microprestress solidification theory for concrete creep. I: Aging and drying effects. Journal of Engineering Mechanics 123:1188-1194, 1997. 
[8] Z. Bažant, S. Baweja. Creep and shrinkage prediction model for analysis and design of concrete structures: Model B3. Adam Neville Symposium: Creep and Shrinkage - Structural Design Effects 2000.

[9] P. Havlásek. Creep and Shrinkage of Concrete Subjected to Variable Environmental Conditions, PhD. Thesis. Czech Technical University in Prague, 2014. 